\title{
Formation control of Multi-agents Based on Method of Matrices
}

\author{
Cheng Zhou ${ }^{1}$, Youqian Feng ${ }^{1}$, Zhonghai Yin ${ }^{1}$, Lei Wang ${ }^{2}$, Xin Zhao ${ }^{3}$ \\ ${ }^{1}$ Science College, Air Force Engineering University, xi'an, China \\ ${ }^{2}$ The second training brigade of People's Liberation Army Air Force Harbin Flight Academy, Harbin, China \\ ${ }^{3}$ Air force early warning academy, Wuhan, China
}

\begin{abstract}
Formation control is one of the most actively studied topics in multi-agents systems, in which all agents will form a desired formation to complete certain tasks. In this paper, a method of matrices is proposed to describe information about formation. Additionally, a flocking algorithm with virtual agents is proposed to control all agent to complete task of desired formation. Simulation results show that all agents can form a desired formation and their velocities consensus are verified.
\end{abstract}

\section{INTRODUCTION}

Flocking is a form of collective behavior of large number of interacting agents with a common group objective to complete certain tasks, and their coordination control has attracted large amounts of research interest ${ }^{[1]}$. OlfatiSaber $^{[2]}$ proposes a theoretical framework for design and analysis of distributed flocking algorithms. It primarily discusses potentials to control agents for free flocking and obstacle avoidance.

Formation control, which is one of the most actively studied topics within the realm of multi-agents systems ${ }^{[3]}$. Recently, a surge of interest has focused on formation control. Oh K, etc. ${ }^{[3]}$ divide the existing research on formation control into three types, position-based, displacement-based, and distance-based for the different types of sensed and controlled variables. It should be note that this categorization is primarily due to the requirement on the sensing capability and interaction topology. Additionally, according to the different methods of control, formation control can be divided into leader-following ${ }^{[4]}$, behavior-based ${ }^{[5,6]}$, virtual structure ${ }^{[7]}$.

In this paper, we propose a method of matrices to describe information of formation, in which reference matrices is used to record the desired graphical information of multi-agents. Additionally, inspired by the distributed flocking algorithm and the method of leaderfollowing, we introduce a flocking algorithm with virtual agents to control multi-agents to form a desired formation.

\section{PROBLEM DESCRIPTION}

Consider a group of $n$ numbered agents moving on the plane, in which the first one $(n=1)$ is "leader", and others numbered by $2,3, \cdots, n$ are "followers". Meanwhile, the motion of leader is independent, other agents of followers will influenced by leader and other followers.

Considering a potential function which is influenced by the neighbors of all agents ${ }^{[1,2]}$, the control protocol $u_{i}$ can be specified as

$$
u_{i}=-k_{p} \sum_{i}^{n} \nabla_{p_{i}} V_{i}-k_{v} \sum_{j \in N_{i}}\left(v_{i}-v_{j}\right)
$$

Where $k_{p}>0, k_{v}>0$ are the parameters of position and velocity. $V_{i}$ is the total potential of agent $i$, which is denoted by

$$
V_{i}=\sum_{j \in N_{i}} V_{i j}\left(\left\|p_{i j}\right\|\right)
$$

Where $V_{i j}$ is a differentiable, nonnegative, function of the distance $\left\|p_{i j}\right\|$ between agents $i$ and $j$.

Thus, the problem of formation can be described as

$$
\begin{aligned}
& \lim _{t \rightarrow \infty}\left(p_{i}-h_{i}-p_{1}\right)=0 \\
& \lim _{t \rightarrow \infty}\left(v_{i}-v_{1}\right)=0, i \in n
\end{aligned}
$$

Where $h_{i}$ is the desired formation from ith agent to leader in a global coordinate system.

\section{THE METHOD OF MATRICES}

\subsection{Reference matrices}

Reference matrices is the fundamental of formation. It is used to record the graph of formation. Thus, the $n \times n$ reference matrices can be formally represented by

\footnotetext{
*Corresponding author: zhou417161659@163.com
} 


$$
F=\left[\begin{array}{cccc}
f_{11} & f_{12} & \cdots & f_{1 n} \\
f_{21} & f_{22} & \cdots & f_{2 n} \\
\vdots & \vdots & \ddots & \vdots \\
f_{n 1} & f_{n 2} & \cdots & f_{n n}
\end{array}\right]
$$

Where $f_{i j}$ is a Boolean variable. 0 means that it does not exist any agent in $f_{i j}$, and vice versa, i.e.,

$$
f_{i j}=\left\{\begin{array}{cc}
1 & \text { agent } \text { exist } \\
0 & \text { others }
\end{array}\right.
$$

\subsection{Calculation of location}
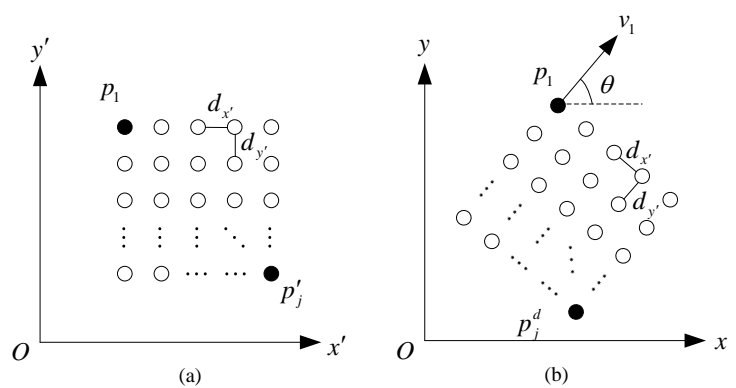

Figure 1. Calculation of descried location

In a $m \times m$ reference matrices, the desired location $p_{j}^{d}=\left(x_{j}^{d}, y_{j}^{d}\right)$ of $j$ th agent can be obtained by the actual location of first agent $p_{1}=\left(x_{1}, y_{1}\right)$ through geometrical relations. The relative coordinate system $x^{\prime} o y^{\prime}$ is constructed by formation, as shown in Fig 1(a). Let the distances of rows and columns be $d_{x^{\prime}}$ and $d_{y^{\prime}}$. Thus, the relative desired location $p_{j}^{\prime}=\left(x_{j}^{\prime}, y_{j}^{\prime}\right)$ of $j$ th agent in relative coordinate system $x^{\prime} o y^{\prime}$ can be calculated by the actual location $p_{1}=\left(x_{1}, y_{1}\right)$, specified as,

$$
\left[\begin{array}{l}
x_{j}^{\prime} \\
y_{j}^{\prime}
\end{array}\right]=\left[\begin{array}{l}
x_{1} \\
y_{1}
\end{array}\right]+\left[\begin{array}{c}
c_{j}-c_{1} \\
r_{1}-r_{j}
\end{array}\right]\left[\begin{array}{l}
d_{x^{\prime}} \\
d_{y^{\prime}}
\end{array}\right]
$$

Where $c_{j}$ and $r_{j}$ are the rows and columns of $j$ th agent in formation matrices. Furthermore, we can obtain the desired location $p_{j}^{d}=\left(x_{j}^{d}, y_{j}^{d}\right)$ of $j$ th agent in global coordinate system xoy through rotation of coordinates, specified as

$$
\left\{\begin{array}{l}
x_{j}^{d}=x_{1}+\left(y_{j}^{\prime}-y_{1}\right) \cos \theta+\left(x_{j}^{\prime}-x_{1}\right) \sin \theta \\
y_{j}^{d}=y_{1}+\left(y_{j}^{\prime}-y_{1}\right) \sin \theta-\left(x_{j}^{\prime}-x_{1}\right) \cos \theta
\end{array}\right.
$$

Where $\theta$ is the angle of velocity $v_{1}$ direction with $x$ -axis. $x_{j}^{\prime}$ and $y_{j}^{\prime}$ are the relative desired location, shown in Fig.1(b).

\section{CONTROL PROTOCOL}

Olfati-Saber ${ }^{[2]}$ designs a distributed flocking algorithm, where those agents in flocking can be divided into two kinds. Some are that consist of flock members, called $\alpha$ agent; The others are virtual agents associated with respective objective for each $\alpha$-agent, called $\gamma$-agent. (As this paper does not relate to obstacle avoidance, we will not discuss the virtual agents of $\beta$-agent). As shown in Fig 2(a), all $\alpha$-agents will arrive at the balance point in the effect of potentials. Meanwhile, the $\gamma$-agent will generate navigation information to guide all $\alpha$-agents to the objective point.
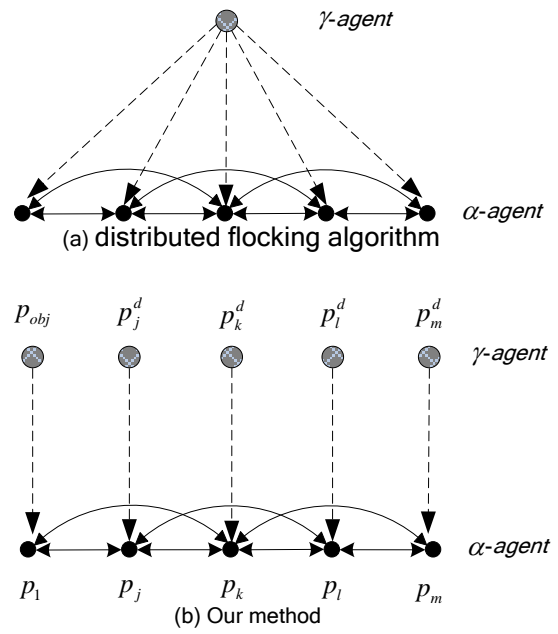

Figure 2. The control protocol of collective potentials

However, the distributed flocking algorithm is not capable of completing a complicated task that form a specific formation from scattered to orderly. Inspired by the distributed flocking algorithm and the model of leader-follower, we introduce an improved flocking algorithm to form formation from scattered to orderly for whole agents. As shown in Fig 2(b), the $\alpha$-agent of our method is same as the distributed flocking algorithm, which can be utilized to remain specific distance and avoid collision between agents. However, the $\gamma$-agent can be divided into two kinds in our method. (1) The $\gamma$ agent of leader is located at the global objective point $p_{o b j}$, which is utilized to guide all $\alpha$-agent to move to the objective collectively. (2) Those rest $\gamma$-agents are located at the desired location $p_{j}^{d}$ of their respective $\alpha$ agents. Thus all agents will construct a intended formation under the whole virtual agents. The control protocol $u_{i}$ of $i$ th agent can be specified as,

$$
u_{i}=u_{i}^{\alpha}+u_{i}^{\gamma}
$$

Where $u_{i}^{\alpha}$ is the control feedback of $i$ th agent from surrounding $\alpha$-agents. $u_{i}^{\gamma}$ is the navigation information of $i$ th agent from its corresponding $\gamma$-agent. The control feedback $u_{i}^{\alpha}$ can be calculated by

$$
u_{i}^{\alpha}=c_{1} \Phi\left(f_{a}^{i}\right)+c_{2} \Phi\left(f_{v c}^{i}\right)+c_{3} \Phi\left(f_{r}^{i}\right)
$$

Where $f_{a}^{i}, f_{v c}^{i}$ and $f_{r}^{i}$ are the attractive force, velocity consensus, and repulsive force of $i$ th agent from its surrounding $\alpha$-agents, respectively. $c_{1}, c_{2}$ and $c_{3}$ are their respective parameters. 
Let $d_{c}$ be the maximum range of communication between $\alpha$-agents, and $a_{i j}$ be the adjacency matrix of $\alpha$-agents. Let $\mathrm{v}$ be the minimum range of attractive force between $\alpha$-agents, and $d_{r}$ be the minimum range of repulsive force between $\alpha$-agents. Thus the scope of velocity consensus is from $d_{r}$ to $d_{a}$. Let $p_{i}$ be the location of $i$ th agent. The location of surrounding agents $j$ are $p_{j}$, and their velocities are $v_{j}$. Thus the corresponding force $f_{a}^{i}, f_{v c}^{i}$ and $f_{r}^{i}$ can be obtained by

$$
\begin{gathered}
f_{a}^{i}=\sum_{j=1}^{n} \frac{1}{2}\left(d_{a}-\left\|p_{i}-p_{j}\right\|\right) * \frac{\left(p_{i}-p_{j}\right)}{\left\|p_{i}-p_{j}\right\|} \\
f_{v c}^{i}=a_{i j} \sum_{j=1}^{n} v_{j} \\
f_{r}^{i}=\sum_{j=1}^{n}\left(\frac{d_{r}}{\left\|p_{i}-p_{j}\right\|}-1\right) * \frac{\left(p_{i}-p_{j}\right)}{\left\|p_{i}-p_{j}\right\|} \\
\Phi(x)=\left(\frac{\|x\|}{\sqrt{1+(\|x\|)^{2}}}\right) \frac{x}{\|x\|}
\end{gathered}
$$

Additionally, we assume that the first agent be the leader to guide other agents to the global objective point. The rest agents will regard their desired location as respective local objective point to follow the leader until the desired formation is constructed. Thus the navigation feedback $u_{i}^{\gamma}$ of agents $i$ can be computed as

$$
u_{i}^{\gamma}=c_{4} \Phi\left(f_{\text {objp }}^{i}\right)+c_{5} \Phi\left(f_{\text {objv }}^{i}\right)
$$

Where $f_{\text {objp }}^{i}$ and $f_{\text {objv }}^{i}$ are the attractive force and velocity consensus of agents $i$, generated by its corresponding $\gamma$-agent. $c_{4}$ and $c_{5}$ are their respective parameters.

(1) For leader: the attractive force $f_{\text {objp }}^{1}$ and velocity consensus $f_{o b j v}^{1}$ from the global objective point can be calculated by

$$
\left\{\begin{array}{l}
f_{o b j p}^{1}=p_{o b j}-p_{1} \\
f_{o b j v}^{1}=v_{o b j}-v_{1}
\end{array} \quad i=1\right.
$$

Where $p_{o b j}$ is the location of global objective point, and $v_{o b j}$ is the desired velocity when reach to $p_{o b j} \cdot p_{1}$ and $v_{1}$ are the actual location and velocity of leader.

(2) For other $\alpha$-agents: the attractive force $f_{\text {objp }}^{i}$ and velocity consensus $f_{o b j v}^{i}$ from desired objective point for agent $i$ can be calculated by

$$
\left\{\begin{array}{c}
f_{o b j p}^{i}=p_{i}^{d}-p_{i} \\
f_{\text {objv }}^{i}=v_{1}-v_{i}
\end{array} \quad i \neq 1\right.
$$

Where $p_{i}^{d}$ is the desired location for agent $i . p_{i}$ and $v_{i}$ are the actual location and velocity for agent $i$.

\section{SIMULATION}

In this section, simulation tests are performed to demonstrate the improved method. In this scenario, several agents construct a formation of number " 1 " in a $8 \times 8$ reference matrices.

Meanwhile, we calculate all desired location for each agent based on leader(the first agent). At last, the control outputs of each agent can be calculated by the strategy of control protocol. Additionally, results of simulation are specified as follows.

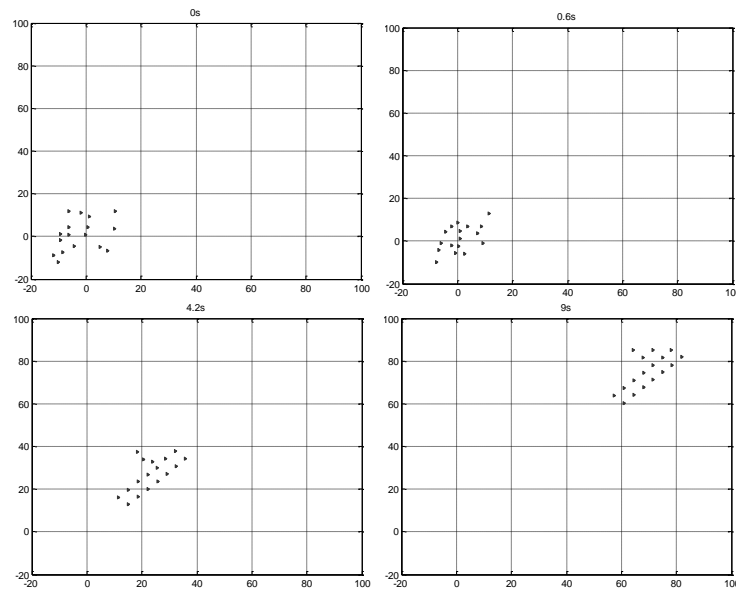

Figure 3. The simulation of formation of number " 1 "

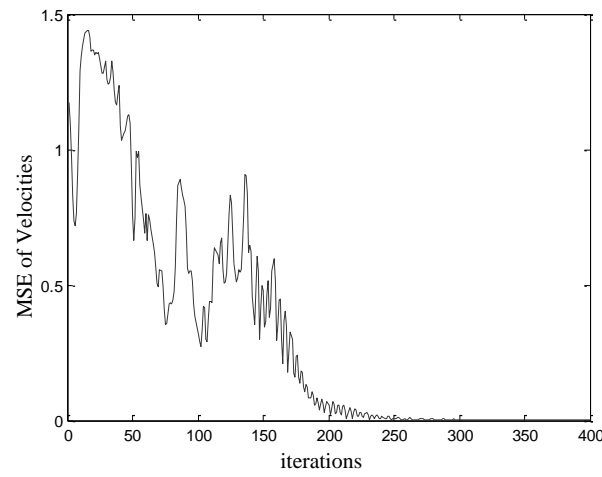

Figure 4. The mean square error of velocities

It can be observed in detail that all agents move towards the global objective point from a scattered formation to a orderly formation of number " 1 ". Additionally, the mean square error (MSE) of velocities of all agents is depicted by Figure 4. It is clear that the MSE of velocities close to 0 at nearly 300 iterations, i.e., the velocity consensus is verified for our method.

\section{CONCLUSIONS}

In this paper, a method of matrices is proposed to describe information of formation. Furthermore, the locations of whole agents can be obtained through matrices in global coordinate system. Inspired by the distributed flocking algorithm and the model of leaderfollower, a flocking algorithm with virtual agents is proposed to form formation from scattered to orderly for multi-agents. At last, a scenario is designed to verify our 
method, in which several agents construct a formation of number "1". Simulation results show that agents will form a desired formation gradually. And all agent will have a consensus velocity.

Future works contain two directions. First, a control protocol is utilized for time-relay or varied topology. Second, a control protocol is designed for obstacle avoidance.

\section{REFERENCES}

1. Tanner H G, Jadbabaie A, Pappas G J. Flocking in fixed and switching networks[J]. IEEE Transactions on Automatic control, 2007, 52(5): 863-868.

2. Olfati-Saber R. Flocking for multi-agent dynamic systems: Algorithms and theory[J]. IEEE Transactions on automatic control, 2006, 51(3): 401-420.

3. Oh K K, Park M C, Ahn H S. A survey of multiagent formation control[J]. Automatica, 2015, 53: 424-440.

4. Ni W, Cheng D. Leader-following consensus of multi-agent systems under fixed and switching topologies[J]. Systems \& Control Letters, 2010, 59(3): 209-217.

5. Xu D, Zhang X, Zhu Z, et al. Behavior-based formation control of swarm robots[J]. Mathematical Problems in Engineering, 2014, 2014.

6. Sen A, Sahoo S R, Kothari M. A cooperative targetcentric formation with bounded acceleration[J]. IFAC-PapersOnLine, 2016, 49(1): 425-430.

7. Low C B. A flexible virtual structure formation keeping control design for nonholonomic mobile robots with low-level control systems, with experiments[C]//Intelligent Control (ISIC), 2014 IEEE International Symposium on. IEEE, 2014: 1576-1582. 